\title{
Finemet Thin Films Substituted by Chromium - CEMS and MOKE Study
}

\author{
K. Brzózka ${ }^{a}$, T. Szumiata ${ }^{a}, \mathrm{P}$. Sovák ${ }^{b}$, B. Górka ${ }^{a}, \mathrm{~K}_{\text {. Hibner }}^{a}$ And J. Balcerski ${ }^{c}$ \\ ${ }^{a}$ Department of Physics, Technical University of Radom, Krasickiego 54, 26-600 Radom, Poland \\ ${ }^{b}$ Institute of Physics, P.J. Šafárik University, Park Angelinum 9, 04154 Košice, Slovakia \\ ${ }^{c}$ Solid State Physics Department, University of Łódź, Pomorska 149/153, 90-236 Łódź, Poland
}

\begin{abstract}
Conversion electron Mössbauer spectroscopy and magneto-optical Kerr effect were applied to investigate hyperfine interactions and macroscopic magnetic properties of Finemet-type thin films. Phase analysis as well as compositional evolution of hyperfine parameters and coercivity was performed in the range of chromium percentage $0 \leq x \leq 14$. Essential differences in structure and hyperfine parameters were found for films of different chromium concentration. Coercivity shows non-monotonic dependence with minimal value at $x=5$.
\end{abstract}

PACS numbers: 75.50.Bb, 61.46.Hk, 76.80.+y, 78.20.Ls

\section{Introduction}

The influence of chromium substitution on structure and some magnetic characteristics of Finemet thin films is the subject of this paper. Finemet alloys of original composition $\mathrm{Fe}_{73.5} \mathrm{Cu}_{1} \mathrm{Nb}_{3} \mathrm{Si}_{13.5} \mathrm{~B}_{9}$ and similar alloys with modified chemical constitution are usually obtained in a form of amorphous ribbons by rapid quenching of melt and optionally they are subjected to the controlled annealing in order to form nanocrystalline structure. The alloys are well-known for excellent soft magnetic properties resulting in many applications [1-4]. Employment of the form of thin film gives additional possibilities, for instance Finemet substituted by Cr can be used for fabrication of magnetic thin film inductors in high-frequency applications [5]. Properties of thin films can differ from the bulk material ones due to another preparation method and considerable fraction of surface regions.

\section{Experimental methods}

$40 \mathrm{~nm}$ thick $\mathrm{Fe}_{73.5-x} \mathrm{Cr}_{x} \mathrm{Cu}_{1} \mathrm{Nb}_{3} \mathrm{Si}_{13.5} \mathrm{~B}_{9}$ films $(x=0$, $1,3,5,9,11,14)$ were deposited upon a glass substrate using DC sputtering method and afterwards they were annealed under vacuum for $1 \mathrm{~h}$ at the temperature $500{ }^{\circ} \mathrm{C}$. The primary samples have rectangular shape with sizes: $2 \mathrm{~cm} \times 3 \mathrm{~cm}$. Phase composition and hyperfine parameters were examined by means of conversion electron Mössbauer spectroscopy (CEMS) using ${ }^{57} \mathrm{Co}(\mathrm{Rh})$ source of gamma radiation and a gas-flow detector supplied with $\mathrm{He}+4 \% \mathrm{CH}_{4}$ mixture. To analyze the Mössbauer spectra, program NORMOS was employed. Hysteresis loops were recorded with magneto-optical Kerr effect (MOKE) system operating in $\mathrm{AC}$ mode. The system consists of a red laser diode, a crystalline polarizer, a half-wave plate, a Wollaston prism, two-photodiode differential detector and a coreless magnetizing coil supplied with $50 \mathrm{~Hz}$ current. Both MOKE signal from detector and Hall voltage from magnetic field sensor were registered by use of an oscilloscope.

\section{Results}

CEMS outcomes reveal compositional evolution of phases in investigated samples (Fig. 1a-c). In the Mössbauer spectra recorded for films of small $\mathrm{Cr}$ concentration $(x<5)$ a discrete component dominates, composed of four or five Zeeman sextets with line width $0.34 \div 0.48 \mathrm{~mm} / \mathrm{s}$ and magnetic hyperfine field values slightly dependent on $x$ (Fig. 2). Besides, a smeared low-field sextet exists in central part of the spectra, with distributed magnetic hyperfine field (MHF) and mean MHF value about $5 \mathrm{~T}$. It originates probably from the amorphous remainder. The discrete component is attributed mainly to bcc Fe-Si phase with possible admixture of $\mathrm{Cr}$. Relative content of this subspectrum amounts to about $80 \%$ at $x=1$ and is strongly reduced with growing $\mathrm{Cr}$ percentage. For $x>5$ it is below the level of statistical noise, so the samples seem to be nearly full amorphous. Instead, development of a subspectrum representing an amorphous phase composed of a smeared high-field sextet as well as a paramagnetic doublet (attributed to regions rich in $\mathrm{Cr}$ ) is observed with increasing $x$. This is in agreement with the results reported for similar bulk alloys [6] where a strong increase of crystallization temperature with rising $x$ was stated. MHF of the amorphous component has the largest value $\langle B\rangle_{\mathrm{am}}=21 \mathrm{~T}$ at chromium concentration $x=5$ and then it is strongly reduced (Fig. 2). As a result, the mean value of MHF (over the whole alloy) decreases almost monotonically from about $26.1 \mathrm{~T}$ to $8.9 \mathrm{~T}$, as shown in Fig. 3 . 


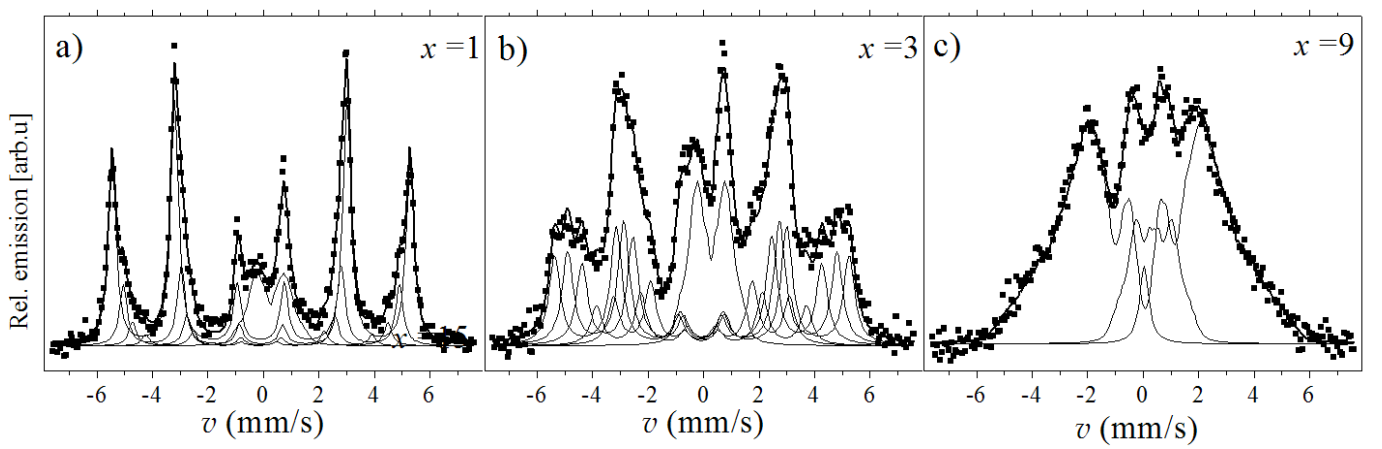

Fig. 1. CEMS spectra recorded for $\mathrm{Fe}_{73.5-x} \mathrm{Cr}_{x} \mathrm{Cu}_{1} \mathrm{Nb}_{3} \mathrm{Si}_{13.5} \mathrm{~B}_{9}$ thin films (annealed at $\left.500{ }^{\circ} \mathrm{C}\right): x=1$ (a), $x=3$ (b), $x=9$ (c).

It has been evaluated (by extrapolation) that the magnetic order in the amorphous matrix diminishes at about $x=18$ at.\%, in agreement with the results obtained for Cr-substituted bulk Finemet-like alloys [7]. All CEMS spectra show similar spin texture with magnetic moments arranged in-plane of the sample.

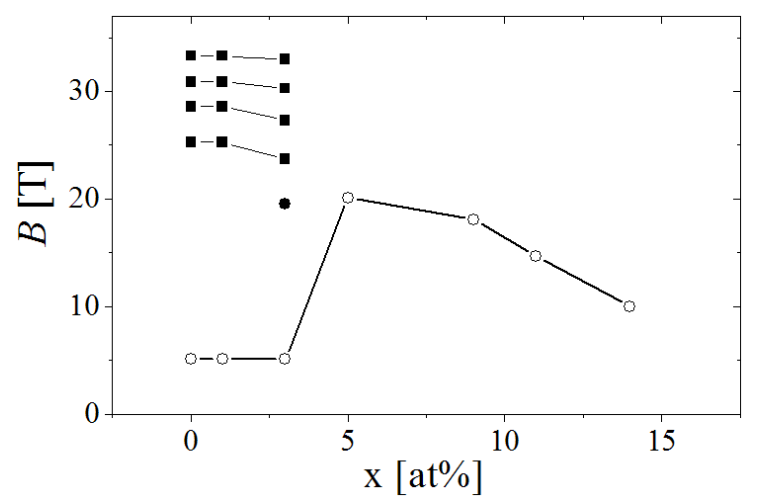

Fig. 2. MHF determined from CEMS spectra of $\mathrm{Fe}_{73.5-x} \mathrm{Cr}_{x} \mathrm{Cu}_{1} \mathrm{Nb}_{3} \mathrm{Si}_{13.5} \mathrm{~B}_{9}$ films (annealed at $500{ }^{\circ} \mathrm{C}$ ): the amorphous component - open circles, discrete sextets - closed symbols.

The shape of magnetic hysteresis loop and its parameters evolve with alloys composition (Fig. 4). Coercivity fluctuates around $1.3 \div 1.7 \mathrm{mT}$ in the concentration range $5 \leq x \leq 14$ and increases strongly up to $11 \mathrm{mT}$ when $x$ decreases to zero. A remarkable feature of the studied films is dependence of the coercivity value as well as the shape of the hysteresis curve on the direction of the magnetization vector in-plane of the film (Fig. 5). For instance, when the sample with $x=14$ is magnetized along the longer side of the sample (magnetization angle $\alpha=0^{\circ}$ ), the hysteresis loop is shaped rectangularly and coercivity is equal to $1.7 \mathrm{mT}$. When the magnetic field is applied parallel to the shorter edge $\left(\alpha=90^{\circ}\right)$, the hysteresis curve becomes more oblique and magnetization saturates slower, however coercive field drops to about $0.5 \mathrm{mT}$. In the investigated films the alignment

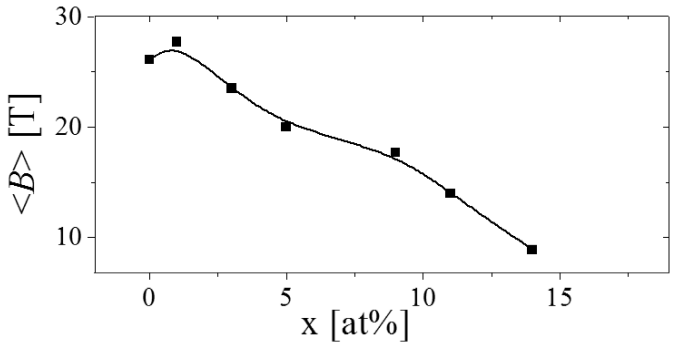

Fig. 3. Mean MHF (over the whole sample) determined from CEMS spectra of $\mathrm{Fe}_{73.5-x} \mathrm{Cr}_{x} \mathrm{Cu}_{1} \mathrm{Nb}_{3} \mathrm{Si}_{13.5} \mathrm{~B}_{9}$ thin films (annealed at $500^{\circ} \mathrm{C}$ ).

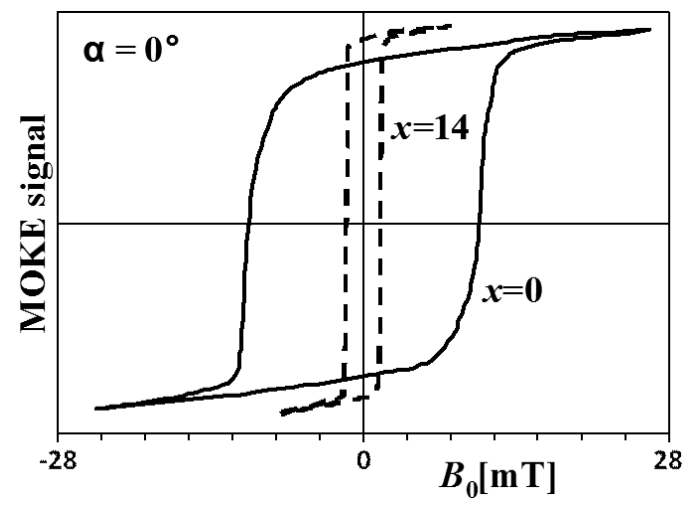

Fig. 4. Hysteresis loops recorded for Finemet thin films of composition $\mathrm{Fe}_{73.5-x} \mathrm{Cr}_{x} \mathrm{Cu}_{1} \mathrm{Nb}_{3} \mathrm{Si}_{13.5} \mathrm{~B}_{9}, x=$ 0,14 (magnetization angle $\alpha=0^{\circ}-$ see text).

of the in-plane magnetic anisotropy axis does not show systematic correlation with the edges of the rectangular substrates. This is connected with conditions of the samples fabrication process which produces internal strain in the films and with different content of bcc Fe iron rich phase. Such a behavior makes difficult precise studies of compositional evolution of the coercive field. 


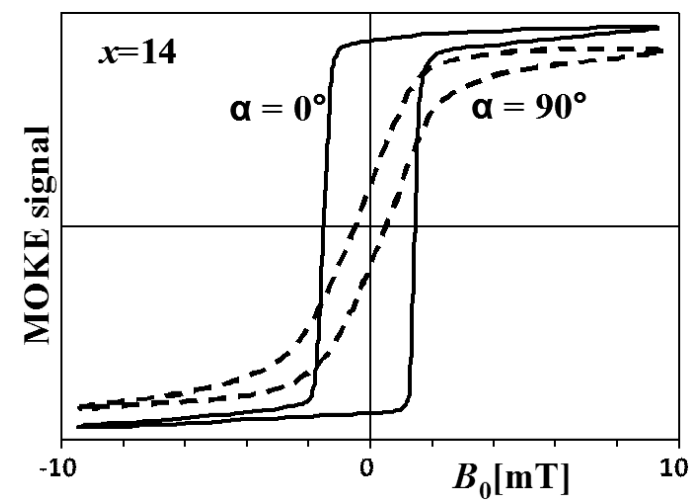

Fig. 5. Hysteresis loops recorded for Finemet thin films of composition $\mathrm{Fe}_{59.5} \mathrm{Cr}_{14} \mathrm{Cu}_{1} \mathrm{Nb}_{3} \mathrm{Si}_{13.5} \mathrm{~B}_{9}$ (magnetization angle $\alpha=0^{\circ}, 90^{\circ}$ - see text).

\section{Conclusions}

The study reveals prevalence of the crystalline phase (bcc Fe-Si) in alloys with chromium concentration $x \leq 3$ and dominance of the amorphous regions in films with $x \geq 5$. It was found that the extent of crystallization and the mean magnetic hyperfine field value decrease with increasing $x$. The random orientation of (in-plane) magnetic anisotropy axis was stated. The minimal value of coercivity $B_{\mathrm{c}}=1.3 \mathrm{mT}$ was found for chromium percentage $x=5$.

\section{Acknowledgments}

The paper was supported by Slovak Grant Agency VEGA $(1 / 0167 / 10)$.

\section{References}

[1] G. Herzer, Phys. Scr. T 49, 307 (1993).

[2] P. Butvin, B. Butvinova, J.M. Silveyra, M. Chromcikova, D. Janickovic, J. Sitek, P. Svec, G. Vlasak, J. Magn. Magn. Mater. 322, 3035 (2010).

[3] J.M. Silveyra, E. Illekova, P. Svec, D. Janickovic, A. Rosales-Rivera, V.J. Cremaschi, Physica B 405, $2720(2010)$.

[4] T. Szumiata, B. Górka, A. Zorkovska, P. Sovak, J. Magn. Magn. Mater. 295, 95 (2005).

[5] Y. Zhou, Z.-M. Zhou, Y. Cao, X.-Y. Gao, W. Ding, J. Magn. Magn. Mater. 320, e963 (2008).

[6] V. Franco, C.F. Conde, A. Conde, B. Varga, A. Lovas, J. Magn. Magn. Mater. 215-216, 404 (2000).

[7] J. Kováč, O. Duša, M. Konč, T. Švec, P. Sovák, J. Magn. Magn. Mater. 157-158, 197 (1996). 